PROCEEDINGS OF THE

AMERICAN MATHEMATICAL SOCIETY

Volume 139, Number 11, November 2011, Pages 4063-4072

S 0002-9939(2011)10975-4

Article electronically published on March 30, 2011

\title{
SCHUR-AGLER CLASS RATIONAL INNER FUNCTIONS ON THE TRIDISK
}

\author{
GREG KNESE
}

(Communicated by Richard Rochberg)

\begin{abstract}
We prove two results with regard to rational inner functions in the Schur-Agler class of the tridisk. Every rational inner function of degree $(n, 1,1)$ is in the Schur-Agler class, and every rational inner function of degree $(n, m, 1)$ is in the Schur-Agler class after multiplication by a monomial of sufficiently high degree.
\end{abstract}

\section{Prologue}

In this article, we continue the study of the Schur-Agler class of the polydisk by focusing on rational inner functions. The Schur-Agler class appears naturally in operator theory as the class of holomorphic functions $f: \mathbb{D}^{n} \rightarrow \mathbb{D}$ which satisfy the von Neumann inequality; i.e. for all commuting $n$-tuples of strict contractions $\left(T_{1}, \ldots, T_{n}\right)$ on some separable Hilbert space, we have

$$
\left\|f\left(T_{1}, \ldots, T_{n}\right)\right\| \leq 1 .
$$

The Schur class simply refers to the holomorphic functions $f: \mathbb{D}^{n} \rightarrow \mathbb{D}$. Our general motivating question is this:

How does the Schur-Agler class fit inside the Schur class?

For $n=1,2$ these two classes coincide, but they differ for $n \geq 3$, and this is not well understood. More recent efforts in this area have focused on generalizations and properties of the Schur-Agler class. See Anderson et al., 2008, Ball and Bolotnikov, 2002, Ball and Bolotnikov, 2010. For more specific progress on this question, one probably has to go back to work of the 1970's on counterexamples to von Neumann's inequality. See Varopoulos, 1974], Crabb and Davie, 1975, Lotto, 1994, Holbrook, 2001.

Motivated by the recent major strides in the study of two variable rational inner functions from Cole and Wermer, 1999, Geronimo and Woerdeman, 2004, Ball et al., 2005, along with our own efforts Knese, 2008, Knese, 2010a, the approach of this article is to make progress on this question by studying rational inner functions in the Schur-Agler class on $\mathbb{D}^{3}$. For further motivation and background to this approach we refer the reader to Knese, 2010b and Knese, 2010c. We now

Received by the editors October 5, 2010.

2010 Mathematics Subject Classification. Primary 47A57; Secondary 42B05.

Key words and phrases. Schur-Agler class, von Neumann's inequality, bidisk, polydisk, rational inner function, trigonometric polynomials, sums of squares.

This research was supported by NSF grant DMS-1048775.

(C)2011 American Mathematical Society 
introduce our topic purely in terms of polynomials, as our main results serve to establish a close connection between sums of squares decompositions for positive trigonometric polynomials and the Schur-Agler class on the tridisk $\mathbb{D}^{3}$.

\section{Rational inner functions in the Schur-Agler Class}

Let $\mathbb{D}, \mathbb{D}^{n}, \mathbb{T}, \mathbb{T}^{n}$ denote the unit disk, polydisk, torus, and $n$-torus. We say $p \in \mathbb{C}\left[z_{1}, \ldots, z_{n}\right]$ has multi-degree at most $\mathbf{d}=\left(d_{1}, \ldots, d_{n}\right)$ if it has degree at most $d_{j}$ in the variable $z_{j}$.

If $p$ has multi-degree at most $\mathbf{d}$ we may form a type of reflection (depending on the degree)

$$
\tilde{p}(z):=z^{\mathbf{d}} \overline{p\left(1 / \bar{z}_{1}, \ldots, 1 / \bar{z}_{n}\right)} \in \mathbb{C}\left[z_{1}, \ldots, z_{n}\right],
$$

and if in addition $p$ has no zeros on $\mathbb{D}^{n}$, then the rational function

$$
\phi(z)=\frac{\tilde{p}(z)}{p(z)}
$$

is a rational inner function; i.e. has modulus one a.e. on $\mathbb{T}^{n}$ and modulus at most one on $\mathbb{D}^{n}$, by the maximum principle. Theorem 5.2.5 of Rudin, 1969 proves that every rational inner function on $\mathbb{D}^{n}$ arises as in (2.1).

In particular,

$$
\begin{aligned}
& |p(z)|^{2}-|\tilde{p}(z)|^{2}=0 \text { on } \mathbb{T}^{n}, \\
& |p(z)|^{2}-|\tilde{p}(z)|^{2} \geq 0 \text { on } \overline{\mathbb{D}}^{n} .
\end{aligned}
$$

On the other hand, any expression of the form

$$
\sum_{j=1}^{n}\left(1-\left|z_{j}\right|^{2}\right) \operatorname{SOS}_{j}(z),
$$

where each $S O S_{j}$ is a sum of squared moduli of polynomials, also satisfies this inequality. It turns out that $\tilde{p} / p$ is in the Schur-Agler class exactly when the left side of (2.2) is of the form (2.3).

Theorem 2.1. Given a polynomial $p \in \mathbb{C}\left[z_{1}, \ldots, z_{n}\right]$ with no zeros in $\mathbb{D}^{n}$ and degree at most $\mathbf{d}, \tilde{p} / p$ is in the Schur-Agler class exactly when there exists a decomposition

$$
|p(z)|^{2}-|\tilde{p}(z)|^{2}=\sum_{j=1}^{n}\left(1-\left|z_{j}\right|^{2}\right) \operatorname{SOS}_{j}(z),
$$

where each $\mathrm{SOS}_{j}$ is a sum of squared moduli of polynomials.

This theorem is implicit in Cole and Wermer, 1999. We refined the above theorem as follows.

Theorem 2.2 ([Knese, 2010b]). If $p \in \mathbb{C}\left[z_{1}, \ldots, z_{n}\right]$ has multi-degree at most $\mathbf{d}=$ $\left(d_{1}, \ldots, d_{n}\right)$ and $\tilde{p} / p$ is in the Schur-Agler class, then given a decomposition

$$
|p(z)|^{2}-|\tilde{p}(z)|^{2}=\sum_{j=1}^{n}\left(1-\left|z_{j}\right|^{2}\right) K_{j}(z, z),
$$

where each $K_{j}$ is a positive semi-definite function, it must be the case that $K_{j}$ is a sum of squares of polynomials of degree at most

$$
\left\{\begin{array}{l}
d_{j}-1 \text { in } z_{j}, \\
d_{k} \text { in } z_{k}
\end{array} \text { for } k \neq j .\right.
$$


In particular, $K_{j}$ can be written as a sum of at most $d_{j} \prod_{k \neq j}\left(d_{k}+1\right)$ polynomials (by dimensionality).

Recall that a function $K(z, \zeta)$ is positive semi-definite if for every finite set $F$ the matrix

$$
(K(z, \zeta))_{z, \zeta \in F}
$$

is positive semi-definite. (We would need an ordering to form an actual matrix, but this is unimportant.) For more information on positive semi-definite kernels, refer to Agler and McCarthy, 2002, Section 2.7.

The main results of this paper relate to rational inner functions in the SchurAgler class on $\mathbb{D}^{3}$. The first interesting result in this area is due to Kummert.

Theorem 2.3 (Kummert, 1989a ). If $p \in \mathbb{C}\left[z_{1}, z_{2}, z_{3}\right]$ has degree $(1,1,1)$ and has no zeros on $\overline{\mathbb{D}}^{3}$, then $\tilde{p} / p$ is in the Schur-Agler class.

We gave the following minor improvement to the details of the sums of squares decomposition of $\tilde{p} / p$ in Knese, 2010c.

Theorem 2.4. If $p \in \mathbb{C}\left[z_{1}, z_{2}, z_{3}\right]$ has degree $(1,1,1)$ and no zeros on $\overline{\mathbb{D}}^{3}$, then there exist sums of squares terms such that

$$
|p|^{2}-|\tilde{p}|^{2}=\sum_{j=1}^{3}\left(1-\left|z_{j}\right|^{2}\right) \operatorname{SOS}_{j}(z),
$$

where $\mathrm{SOS}_{3}$ is a sum of two squares, while $S O S_{1}, S O S_{2}$ are sums of four squares.

Our two main results are the following. We extend the above results to the case of polynomials of degree $(n, m, 1)$ and exhibit a new phenomenon in the study of the Schur-Agler class.

Theorem 2.5. If $p \in \mathbb{C}\left[z_{1}, z_{2}, z_{3}\right]$ has degree $(n, 1,1)$ and no zeros on $\overline{\mathbb{D}}^{3}$, then $\tilde{p} / p$ is in the Schur-Agler class. Furthermore, we have a decomposition

$$
|p|^{2}-|\tilde{p}|^{2}=\sum_{j=1}^{3}\left(1-\left|z_{j}\right|^{2}\right) S O S_{j}(z),
$$

where $\mathrm{SOS}_{3}$ is a sum of two squares, while $S O S_{1}, S O S_{2}$ are sums of $4(n-1)$, $2(n+1)$ squares respectively.

We are not claiming that the bounds of $4(n-1)$ and $2(n+1)$ are best possible.

Theorem 2.6. If $p \in \mathbb{C}\left[z_{1}, z_{2}, z_{3}\right]$ has no zeros on $\overline{\mathbb{D}}^{3}$ and degree at most $(n, m, 1)$, then there exist integers $r, s \geq 0$ such that

$$
\frac{z_{1}^{r} z_{2}^{s} \tilde{p}\left(z_{1}, z_{2}, z_{3}\right)}{p\left(z_{1}, z_{2}, z_{3}\right)}
$$

is in the Schur-Agler class.

This phenomenon has not been observed in the study of the Schur-Agler class (although it is analogous to results in "sums of squares" such as Quillen's theorem Quillen, 1968). We do not yet have an example of $p$ such that $\tilde{p} / p$ is not SchurAgler while $z_{1}^{r} z_{2}^{s} \tilde{p} / p$ is Schur-Agler. However, in the last section we explain how a construction might proceed.

We will first discuss two necessary preliminary results and then proceed to the proof of Theorems 2.5 and 2.6 . 


\section{Preliminary Results}

The following result is proven in Megretski, 2003, Dritschel, 2004, Geronimo and Lai, 2006, and [Dumitrescu, 2007].

Theorem 3.1. Suppose $t$ is a d variable, positive trigonometric polynomial:

$$
t(z)=\sum_{-N \leq|\alpha| \leq N} t_{\alpha} z^{\alpha}>0 \text { for all } z=\left(z_{1}, \ldots, z_{d}\right) \in \mathbb{T}^{d},
$$

where we use multi-index notation with $\alpha \in \mathbb{Z}^{d}$. Then $t$ can be written as a sum of squares of polynomials; i.e. there exist $A_{j} \in \mathbb{C}\left[z_{1}, \ldots, z_{d}\right]$ such that

$$
t(z)=\sum_{j=1}^{M}\left|A_{j}(z)\right|^{2} \quad\left(z \in \mathbb{T}^{n}\right) .
$$

Known proofs of this result require both strict positivity and can only control the numbers of polynomials (and their degrees) in the sums of squares decomposition in terms of a bound below on $t$. See Geronimo and Lai, 2006 for more details. It is this subtlety that creates the need to multiply by a sufficiently high degree monomial in Theorem 2.6. We get around this in Theorem 2.5] via the following lemma.

Lemma 3.2. Let $t$ be a non-negative, two variable trigonometric polynomial of degree $(n, 1)$, i.e.

$$
t\left(z_{1}, z_{2}\right)=t_{0}\left(z_{1}\right)+z_{2} t_{1}\left(z_{1}\right)+\overline{z_{2} t_{1}\left(z_{1}\right)} \geq 0,
$$

where $t_{0}, t_{1}$ are one variable trigonometric polynomials of degree at most $n$. Then, there exist $A_{1}, A_{2} \in \mathbb{C}\left[z_{1}, z_{2}\right]$ of degree at most $(n, 1)$ such that

$$
t(z)=\left|A_{1}(z)\right|^{2}+\left|A_{2}(z)\right|^{2} \quad\left(z \in \mathbb{T}^{2}\right) .
$$

This lemma is implicitly known, but in a different language and/or context (and with more complicated proofs and less detail). In Gabardo, 1998 and Bakonyi and Naevdal, 1998, it is proven and phrased in the language of trigonometric moment problems. The connection to sums of squares is because of the main result of Rudin, 1963, which, loosely speaking, says that given a subset $\Lambda$ of $\mathbb{Z}_{+}^{d}$, one can solve (truncated) trigonometric moment problems on $\Lambda-\Lambda$ if and only if non-negative trigonometric polynomials with Fourier support in $\Lambda-\Lambda$ are sums of squares of polynomials with coefficient support in $\Lambda$.

Proof. The proof is really the same as the degree $(1,1)$ case, which we gave in Knese, 2010c. By minimizing (3.1) over $z_{2}$, we see that $t_{0}\left(z_{1}\right) \geq 2\left|t_{1}\left(z_{1}\right)\right|$. This implies that the $2 \times 2$ matrix trigonometric polynomial

$$
T\left(z_{1}\right)=\left[\begin{array}{ll}
\frac{1}{2} t_{0}\left(z_{1}\right) & t_{1}\left(z_{1}\right) \\
\overline{t_{1}\left(z_{1}\right)} & \frac{1}{2} t_{0}\left(z_{1}\right)
\end{array}\right]
$$

is positive semi-definite. By the matrix Fejér-Riesz theorem (due to M. Rosenblum; see Dritschel, 2004 for a recent proof and references), $T$ can be factored as

$$
T\left(z_{1}\right)=A\left(z_{1}\right)^{*} A\left(z_{1}\right),
$$


where $A \in \mathbb{C}^{2 \times 2}\left[z_{1}\right]$ is a matrix polynomial of degree at most $n$. Then,

$$
t\left(z_{1}, z_{2}\right)=\left[\begin{array}{ll}
1 & \bar{z}_{2}
\end{array}\right] T\left(z_{1}\right)\left[\begin{array}{c}
1 \\
z_{2}
\end{array}\right]=\left\|A\left(z_{1}\right)\left[\begin{array}{c}
1 \\
z_{2}
\end{array}\right]\right\|^{2}
$$

is a sum of two squares of the desired type.

\section{Proof of Theorems 2.5 and 2.6}

To prove Theorems 2.5 and 2.6 simultaneously we merely need to keep track of whether we are using Lemma 3.2 or Theorem 3.1. A brief notational warning: if $E$ is a column vector of polynomials in the variables $z_{1}, z_{2}$ (as will occur below), we shall write $\left\|E\left(z_{1}, z_{2}\right)\right\|^{2}$ for the sum of squares of the entries of $E$, and often to save space we write $\|E\|^{2}$ for the same expression. These are all pointwise euclidean norms and do not represent any kind of function space norm.

Write $p(z)=a\left(z_{1}, z_{2}\right)+b\left(z_{1}, z_{2}\right) z_{3}$, where $a, b \in \mathbb{C}\left[z_{1}, z_{2}\right]$ have degree at most $(n, m)$. For $z_{1}, z_{2} \in \mathbb{T}$, by direct computation

$$
|p|^{2}-|\tilde{p}|^{2}=\left(1-\left|z_{3}\right|^{2}\right)\left(\left|a\left(z_{1}, z_{2}\right)\right|^{2}-\left|b\left(z_{1}, z_{2}\right)\right|^{2}\right) .
$$

Then, for $\left(z_{1}, z_{2}\right) \in \mathbb{T}^{2},\left|a\left(z_{1}, z_{2}\right)\right|^{2}-\left|b\left(z_{1}, z_{2}\right)\right|^{2}$ is a non-negative two variable trigonometric polynomial of degree at most $(n, m)$. As $p$ has no zeros on $\overline{\mathbb{D}}^{3},|a|^{2}-$ $|b|^{2}$ is in fact strictly positive on $\mathbb{T}^{2}$, since a zero $\left(z_{1}^{\prime}, z_{2}^{\prime}\right)$ would imply $\left|p\left(z_{1}^{\prime}, z_{2}^{\prime}, \cdot\right)\right|=$ $\left|\tilde{p}\left(z_{1}^{\prime}, z_{2}^{\prime}, \cdot\right)\right|$, and this would mean $z_{3} \mapsto p\left(z_{1}^{\prime}, z_{2}^{\prime}, z_{3}\right)$ has a zero on $\mathbb{T}$.

By Lemma 3.2 or by Theorem 3.1. we may write

$$
\left|a\left(z_{1}, z_{2}\right)\right|^{2}-\left|b\left(z_{1}, z_{2}\right)\right|^{2}=\left\|E\left(z_{1}, z_{2}\right)\right\|^{2} \text { on } \mathbb{T}^{2},
$$

where $E$ is a vector polynomial with values in $\mathbb{C}^{N}$ (this provides a convenient way to represent sums of squares). In the degree $(n, 1,1)$ case we may take $N=2$, and in the $(n, m, 1)$ case we do not know what $N$ is. Set $\tilde{E}\left(z_{1}, z_{2}\right)=z_{1}^{n+r} z_{2}^{m+s} \overline{E\left(1 / \bar{z}_{1}, 1 / \bar{z}_{2}\right)}$, where we assume $E$ has degree $(n+r, m+s)$. Again, in the case $m=1$, we may take $r=s=0$.

We also remark that since $p$ has no zeros on $\overline{\mathbb{D}}^{3}$, a has no zeros on $\overline{\mathbb{D}}^{2}$. By the maximum principle,

$$
\frac{\tilde{b}\left(z_{1}, z_{2}\right)}{a\left(z_{1}, z_{2}\right)}
$$

is analytic and has modulus strictly less than one since $|b|=|\tilde{b}|$ on $\mathbb{T}^{2}$ and since $|a|>|b|$ on $\mathbb{T}^{2}$. In particular, $a+z_{1}^{r} z_{2}^{s} \tilde{b}$ has no zeros on $\overline{\mathbb{D}}^{2}$.

We may polarize formula (4.1) and get for $z_{1}, z_{2} \in \mathbb{T}$,

$$
p\left(z_{1}, z_{2}, z_{3}\right) \overline{p\left(z_{1}, z_{2}, \zeta_{3}\right)}-\tilde{p}\left(z_{1}, z_{2}, z_{3}\right) \overline{\tilde{p}\left(z_{1}, z_{2}, \zeta_{3}\right)}=\left(1-z_{3} \bar{\zeta}_{3}\right)\left\|E\left(z_{1}, z_{2}\right)\right\|^{2},
$$

for $z_{3}, \zeta_{3} \in \mathbb{C}$, which we rearrange into

$$
\begin{aligned}
& p\left(z_{1}, z_{2}, z_{3}\right) \overline{p\left(z_{1}, z_{2}, \zeta_{3}\right)}+z_{3} \bar{\zeta}_{3}\left\|E\left(z_{1}, z_{2}\right)\right\|^{2} \\
& =\tilde{p}\left(z_{1}, z_{2}, z_{3}\right) \overline{\tilde{p}\left(z_{1}, z_{2}, \zeta_{3}\right)}+\left\|E\left(z_{1}, z_{2}\right)\right\|^{2} .
\end{aligned}
$$

Then, for fixed $z_{1}, z_{2} \in \mathbb{T}$ and for varying $z_{3}$, the map

$$
\left[\begin{array}{c}
p\left(z_{1}, z_{2}, z_{3}\right) \\
z_{3} E\left(z_{1}, z_{2}\right)
\end{array}\right] \mapsto\left[\begin{array}{c}
z_{1}^{r} z_{2}^{s} \tilde{p}\left(z_{1}, z_{2}, z_{3}\right) \\
E\left(z_{1}, z_{2}\right)
\end{array}\right]
$$

gives a well-defined isometry $V\left(z_{1}, z_{2}\right)$ (which depends on $z_{1}, z_{2}$ ) from the span of the elements on the left to the span of the elements on the right (the span is taken 
over the above vectors as $z_{3}$ varies). More concretely, by examining coefficients of $z_{3}$, we map

$$
\left[\begin{array}{c}
a\left(z_{1}, z_{2}\right) \\
\overrightarrow{0}
\end{array}\right] \mapsto\left[\begin{array}{c}
z_{1}^{r} z_{2}^{s} \tilde{b}\left(z_{1}, z_{2}\right) \\
E\left(z_{1}, z_{2}\right)
\end{array}\right], \quad\left[\begin{array}{c}
b\left(z_{1}, z_{2}\right) \\
E\left(z_{1}, z_{2}\right)
\end{array}\right] \mapsto\left[\begin{array}{c}
z_{1}^{r} z_{2}^{s} \tilde{a}\left(z_{1}, z_{2}\right) \\
\overrightarrow{0}
\end{array}\right]
$$

This is how the "lurking isometry argument" traditionally works. However, $V\left(z_{1}, z_{2}\right)$ does not extend uniquely to define a unitary on $\mathbb{C}^{N+1}$, and we would like to extend $V\left(z_{1}, z_{2}\right)$ so that $V$ is rational in $z_{1}, z_{2}$.

The definition that Kummert gives in the $(1,1,1)$ case works here with a small modification.

Claim 1. Define

$$
V=\frac{1}{a}\left[\begin{array}{cc}
z_{1}^{r} z_{2}^{s} \tilde{b} & \tilde{E}^{t} \\
E & \frac{E \tilde{E}^{t}-a\left(z_{1}^{r} z_{2}^{s} \tilde{a}+b\right) I}{a+z_{1}^{r} z_{2}^{s} \tilde{b}}
\end{array}\right] .
$$

Then, $V$ is holomorphic in $\mathbb{D}^{2}$ and unitary valued on $\mathbb{T}^{2}$, and $V$ satisfies

$$
V\left(z_{1}, z_{2}\right)\left[\begin{array}{c}
p\left(z_{1}, z_{2}, z_{3}\right) \\
z_{3} E\left(z_{1}, z_{2}\right)
\end{array}\right]=\left[\begin{array}{c}
z_{1}^{r} z_{2}^{s} \tilde{p}\left(z_{1}, z_{2}, z_{3}\right) \\
E\left(z_{1}, z_{2}\right)
\end{array}\right]
$$

for $\left(z_{1}, z_{2}\right) \in \mathbb{T}^{2}$ and hence for all $\left(z_{1}, z_{2}\right) \in \overline{\mathbb{D}}^{2}$ by analyticity. (This is just the content of (4.3).)

Proof of Claim 1. First, $V$ is holomorphic since $a$ and $a+z_{1}^{r} z_{2}^{s} \tilde{b}$ have no zeros on $\overline{\mathbb{D}}^{2}$.

Let

Notice that

$$
S\left(z_{1}, z_{2}\right)=\operatorname{span}\left\{\left[\begin{array}{l}
p\left(z_{1}, z_{2}, z_{3}\right) \\
z_{3} E\left(z_{1}, z_{2}\right)
\end{array}\right]: z_{3} \in \mathbb{C}\right\}
$$

$$
S\left(z_{1}, z_{2}\right)=\operatorname{span}\left\{\left[\begin{array}{c}
z_{1}^{r} z_{2}^{s} \tilde{p}\left(z_{1}, z_{2}, z_{3}\right) \\
E\left(z_{1}, z_{2}\right)
\end{array}\right]: z_{3} \in \mathbb{C}\right\} .
$$

This follows from looking at coefficients of $z_{3}$ as

$$
S\left(z_{1}, z_{2}\right)=\operatorname{span}\left\{\left[\begin{array}{c}
a\left(z_{1}, z_{2}\right) \\
\overrightarrow{0}
\end{array}\right],\left[\begin{array}{l}
b\left(z_{1}, z_{2}\right) \\
E\left(z_{1}, z_{2}\right)
\end{array}\right]\right\}=\operatorname{span}\left\{\left[\begin{array}{l}
1 \\
\overrightarrow{0}
\end{array}\right],\left[\begin{array}{c}
0 \\
E\left(z_{1}, z_{2}\right)
\end{array}\right]\right\},
$$

and one can similarly show that

$$
\operatorname{span}\left\{\left[\begin{array}{c}
z_{1}^{r} z_{2}^{s} \tilde{p}\left(z_{1}, z_{2}, z_{3}\right) \\
E\left(z_{1}, z_{2}\right)
\end{array}\right]: z_{3} \in \mathbb{C}\right\}=\operatorname{span}\left\{\left[\begin{array}{l}
1 \\
\overrightarrow{0}
\end{array}\right],\left[\begin{array}{c}
0 \\
E\left(z_{1}, z_{2}\right)
\end{array}\right]\right\} .
$$

The goal is to show that $V\left(z_{1}, z_{2}\right)$ is a unitary by verifying (4.5), which shows that $V\left(z_{1}, z_{2}\right)$ is isometric on the subspace $S\left(z_{1}, z_{2}\right)$, and by showing that $V\left(z_{1}, z_{2}\right)$ maps $S\left(z_{1}, z_{2}\right)^{\perp}$ isometrically into itself.

To show (4.5) we first observe that

$$
\begin{aligned}
\tilde{E}\left(z_{1}, z_{2}\right)^{t} E\left(z_{1}, z_{2}\right) & =z_{1}^{n+r} z_{2}^{m+s}\left\|E\left(z_{1}, z_{2}\right)\right\|^{2} \\
& =z_{1}^{n+r} z_{2}^{m+s}\left(\left|a\left(z_{1}, z_{2}\right)\right|^{2}-\left|b\left(z_{1}, z_{2}\right)\right|^{2}\right)=z_{1}^{r} z_{2}^{s}(a \tilde{a}-b \tilde{b}),
\end{aligned}
$$

where $a, b$ are reflected at degree $(n, m)$ to give $\tilde{a}, \tilde{b}$.

Here are the computations used to show (4.4) (which is equivalent to (4.5)):

$$
V\left[\begin{array}{l}
a \\
\overrightarrow{0}
\end{array}\right]=\left[\begin{array}{c}
z_{1}^{r} z_{2}^{r} \tilde{b} \\
E
\end{array}\right]
$$


and

$$
V\left[\begin{array}{c}
b \\
E
\end{array}\right]=\frac{1}{a}\left[\begin{array}{c}
z_{1}^{r} z_{2}^{s} \tilde{b} b+z_{1}^{r} z_{2}^{s}(a \tilde{a}-b \tilde{b}) \\
\frac{b\left(a+z_{1}^{r} z_{2}^{s} \tilde{b}\right)+z_{1}^{r} z_{2}^{s}(a \tilde{a}-b \tilde{b})-a\left(z_{1}^{r} z_{2}^{s} \tilde{a}+b\right)}{a+z_{1}^{r} z_{2}^{s} \tilde{b}}
\end{array}\right]=\left[\begin{array}{c}
z_{1}^{r} z_{2}^{s} \tilde{a} \\
\overrightarrow{0}
\end{array}\right] .
$$

Now we show that $V\left(z_{1}, z_{2}\right)$, viewed as a linear map, is isometric on the orthogonal complement of $S\left(z_{1}, z_{2}\right)$. Set $X\left(z_{1}, z_{2}\right)$ equal to the orthogonal complement of $S\left(z_{1}, z_{2}\right)$ in $\mathbb{C}^{1+N}$, and observe that

$$
X\left(z_{1}, z_{2}\right)=\left\{\left[\begin{array}{l}
0 \\
v
\end{array}\right]: v \perp E\left(z_{1}, z_{2}\right)\right\} .
$$

Notice that $v \perp E\left(z_{1}, z_{2}\right)$ if $\tilde{E}^{t} v=0$.

Let us observe what the definition of $V$ does to elements of $X$. For $\vec{x}=\left[\begin{array}{l}0 \\ v\end{array}\right] \in X$, we have

$$
V \vec{x}=\left[\begin{array}{c}
0 \\
-\frac{z_{1}^{r} z_{2}^{s} \tilde{a}+b}{a+z_{1}^{r} z_{2}^{s} \tilde{b}} v
\end{array}\right]=-\frac{z_{1}^{r} z_{2}^{s} \tilde{a}+b}{a+z_{1}^{r} z_{2}^{s} \tilde{b}} \vec{x} .
$$

So, every element of $X$ is an eigenvector with eigenvalue $-\frac{z_{1}^{r} z_{2}^{s} \tilde{a}+b}{a+z_{1}^{r} z_{2}^{s} \tilde{b}}$. This number is unimodular for $\left(z_{1}, z_{2}\right) \in \mathbb{T}^{2}$. This proves $V\left(z_{1}, z_{2}\right)$ is unitary valued, and the claim is proved.

This means $V$ is an $(N+1) \times(N+1)$ two-variable rational matrix-valued inner function. It was proved in Kummert, 1989b (see also Ball et al., 2005) that such functions have transfer function representations. Namely, there exists a $((N+1)+$ $\left.n_{1}+n_{2}\right) \times\left((N+1)+n_{1}+n_{2}\right)$ block unitary

$$
U=\left[\begin{array}{cc}
A & B \\
C & D
\end{array}\right]=\left[\begin{array}{ccc}
A & B_{1} & B_{2} \\
C_{1} & D_{11} & D_{12} \\
C_{2} & D_{21} & D_{22}
\end{array}\right]
$$

where $A$ is an $(N+1) \times(N+1)$ matrix, $B$ is an $(N+1) \times\left(n_{1}+n_{2}\right), C$ is an $\left(n_{1}+n_{2}\right) \times(N+1)$, and $D$ is an $\left(n_{1}+n_{2}\right) \times\left(n_{1}+n_{2}\right)$ (all subdivided as indicated) such that

$$
V\left(z_{1}, z_{2}\right)=A+B d\left(z_{1}, z_{2}\right)\left(I-D d\left(z_{1}, z_{2}\right)\right)^{-1} C
$$

where

$$
d\left(z_{1}, z_{2}\right)=\left[\begin{array}{cc}
z_{1} I_{1} & 0 \\
0 & z_{2} I_{2}
\end{array}\right] .
$$

Here $I_{1}, I_{2}$ are the $n_{1}, n_{2}$-dimensional identity matrices, respectively.

Such a representation is equivalent to the formula

$$
U\left[\begin{array}{c}
I \\
z_{1} G_{1}\left(z_{1}, z_{2}\right) \\
z_{2} G_{2}\left(z_{1}, z_{2}\right)
\end{array}\right]=\left[\begin{array}{c}
V\left(z_{1}, z_{2}\right) \\
G_{1}\left(z_{1}, z_{2}\right) \\
G_{2}\left(z_{1}, z_{2}\right)
\end{array}\right],
$$

where $G_{1}, G_{2}$ are $\mathbb{C}^{n_{1} \times(N+1)}$-, $\mathbb{C}^{n_{2} \times(N+1)}$-valued functions given by

$$
\left[\begin{array}{l}
G_{1}\left(z_{1}, z_{2}\right) \\
G_{2}\left(z_{1}, z_{2}\right)
\end{array}\right]=\left(I-D d\left(z_{1}, z_{2}\right)\right)^{-1} C
$$

Indeed, one can use formula (4.7) to explicitly solve for $V$ as in (4.6). 
Define

$$
Y=\left[\begin{array}{c}
p \\
z_{3} E
\end{array}\right] \text { and } H_{j}=G_{j} Y \text { for } j=1,2 .
$$

Then, by these definitions

$$
U\left[\begin{array}{c}
I \\
z_{1} G_{1} \\
z_{2} G_{2}
\end{array}\right] Y=U\left[\begin{array}{c}
Y \\
z_{1} G_{1} Y \\
z_{2} G_{2} Y
\end{array}\right]=U\left[\begin{array}{c}
p \\
z_{3} E \\
z_{1} H_{1} \\
z_{2} H_{2}
\end{array}\right]
$$

and

$$
\left[\begin{array}{c}
V \\
G_{1} \\
G_{2}
\end{array}\right] Y=\left[\begin{array}{c}
V Y \\
H_{1} \\
H_{2}
\end{array}\right]=\left[\begin{array}{c}
z_{1}^{r} z_{2}^{s} \tilde{p} \\
E \\
H_{1} \\
H_{2}
\end{array}\right]
$$

where the last equality follows from (4.5).

By (4.7) we now have

$$
U\left[\begin{array}{c}
p \\
z_{3} E \\
z_{1} H_{1} \\
z_{2} H_{2}
\end{array}\right]=\left[\begin{array}{c}
z_{1}^{r} z_{2}^{s} \tilde{p} \\
E \\
H_{1} \\
H_{2}
\end{array}\right]
$$

and since $U$ is a unitary we have

$$
\begin{aligned}
& |p|^{2}+\left|z_{3}\right|^{2}\|E\|^{2}+\left|z_{1}\right|^{2}\left\|H_{1}\right\|^{2}+\left|z_{2}\right|^{2}\left\|H_{2}\right\|^{2} \\
& =\left|z_{1}^{r} z_{2}^{s} \tilde{p}\right|^{2}+\|E\|^{2}+\left\|H_{1}\right\|^{2}+\left\|H_{2}\right\|^{2},
\end{aligned}
$$

which can be rearranged to give

$$
|p|^{2}-\left|z_{1}^{r} z_{2}^{s} \tilde{p}\right|^{2}=\sum_{j=1,2}\left(1-\left|z_{j}\right|^{2}\right)\left\|H_{j}\right\|^{2}+\left(1-\left|z_{3}\right|^{2}\right)\|E\|^{2} .
$$

Even though we have not verified that $H_{1}$ and $H_{2}$ are polynomials, this is enough to prove that $z_{1}^{r} z_{2}^{s} \tilde{p} / p$ is in the Schur-Agler class. In fact, Theorem 2.2 forces $\left\|H_{1}\right\|^{2}$, $\left\|H_{2}\right\|^{2}$ to be sums of squares of polynomials of multi-degree $(n+r-1, m+s, 1)$, $(n+r, m+s-1,1)$.

In the case $m=1$, we have $r=s=0, E$ of degree $(n, 1,0)$, and $E$ has values in $\mathbb{C}^{2}$. So, $\|E\|^{2}$ is a sum of two squares, and by dimensional considerations $\left\|H_{1}\right\|^{2}$ is a sum of at most $4(n-1)$ squares and $\left\|H_{2}\right\|^{2}$ is a sum of at most $2(n+1)$ squares. This concludes the proof of Theorems 2.5 and 2.6.

\section{How to COnstruct examples}

How might one construct an example of a rational inner function $\tilde{p} / p$ with $p$ of degree $(n, m, 1)$ and the property that $\tilde{p} / p$ is not in the Schur-Agler class while $z_{1}^{r} z_{2}^{s} \tilde{p} / p$ is? Examining the above proof, it is a matter of choosing $a, b \in \mathbb{C}\left[z_{1}, z_{2}\right]$ such that (1) $a$ has no zeros on $\mathbb{D}^{2},(2)|a|^{2}-|b|^{2} \geq 0$ on $\mathbb{T}^{2}$, and (3) $|a|^{2}-|b|^{2}$ is not a sum of squares of polynomials of degree $(n, m)$. Positive trigonometric polynomials of degree $(n, m)$ which cannot be written as a sum of squares of polynomials of degree $(n, m)$ do exist (see Dritschel, 2004 and Dumitrescu, 2007), so our problem reduces to finding such a trigonometric polynomial of the form $|a|^{2}-|b|^{2}$. We leave this for future work (or future authors). 


\section{ACKNOWLEDGMENT}

The author sincerely thanks the referee for a close reading and a timely report.

\section{REFERENCES}

[Agler and McCarthy, 2002] Agler, J. and McCarthy, J. E. (2002). Pick interpolation and Hilbert function spaces, volume 44 of Graduate Studies in Mathematics. American Mathematical Society, Providence, RI. MR.1882259 (2003b:47001)

[Anderson et al., 2008] Anderson, J. M., Dritschel, M. A., and Rovnyak, J. (2008). Schwarz-Pick inequalities for the Schur-Agler class on the polydisk and unit ball. Comput. Methods Funct. Theory, 8(1-2):339-361. MR2419482 (2009c:47027)

[Bakonyi and Naevdal, 1998] Bakonyi, M. and Naevdal, G. (1998). On the matrix completion method for multidimensional moment problems. Acta Sci. Math. (Szeged), 64(3-4):547-558. MR:1666043 (99k:42020)

[Ball and Bolotnikov, 2002] Ball, J. A. and Bolotnikov, V. (2002). A tangential interpolation problem on the distinguished boundary of the polydisk for the Schur-Agler class. J. Math. Anal. Appl., 273(2):328-348. MR.1932492 (2003i:47016)

[Ball and Bolotnikov, 2010] Ball, J. A. and Bolotnikov, V. (2010). Canonical de Branges-Rovnyak model transfer-function realization for multivariable Schur-class functions. In Hilbert spaces of analytic functions, volume 51 of CRM Proc. Lecture Notes, pages 1-40. Amer. Math. Soc., Providence, RI. MR 2648864

[Ball et al., 2005] Ball, J. A., Sadosky, C., and Vinnikov, V. (2005). Scattering systems with several evolutions and multidimensional input/state/output systems. Integral Equations $O p$ erator Theory, 52(3):323-393. MR2184571 (2006h:47013)

[Cole and Wermer, 1999] Cole, B. J. and Wermer, J. (1999). Andô's theorem and sums of squares. Indiana Univ. Math. J., 48(3):767-791. MR1736979 (2000m:47014)

[Crabb and Davie, 1975] Crabb, M. J. and Davie, A. M. (1975). von Neumann's inequality for Hilbert space operators. Bull. London Math. Soc., 7:49-50. MR0365179 (51:1432)

[Dritschel, 2004] Dritschel, M. A. (2004). On factorization of trigonometric polynomials. Integral Equations Operator Theory, 49(1):11-42. MR2057766 (2005d:47034)

[Dumitrescu, 2007] Dumitrescu, B. (2007). Positive trigonometric polynomials and signal processing applications. Signals and Communication Technology. Springer, Dordrecht. MR 2309555 (2007m:94044)

[Gabardo, 1998] Gabardo, J.-P. (1998). Trigonometric moment problems for arbitrary finite subsets of $\mathbf{Z}^{n}$. Trans. Amer. Math. Soc., 350(11):4473-4498. MR1443194 (99a:42005)

[Geronimo and Lai, 2006] Geronimo, J. S. and Lai, M.-J. (2006). Factorization of multivariate positive Laurent polynomials. J. Approx. Theory, 139(1-2):327-345. MR 2220044 (2007a:47023)

[Geronimo and Woerdeman, 2004] Geronimo, J. S. and Woerdeman, H. J. (2004). Positive extensions, Fejér-Riesz factorization and autoregressive filters in two variables. Ann. of Math. (2), 160(3):839-906. MR2144970(2006b:42036)

[Holbrook, 2001] Holbrook, J. A. (2001). Schur norms and the multivariate von Neumann inequality. In Recent advances in operator theory and related topics (Szeged, 1999), volume 127 of Oper. Theory Adv. Appl., pages 375-386. Birkhäuser, Basel. MR1902811 (2003e:47016)

[Knese, 2008] Knese, G. (2008). Bernstein-Szegö measures on the two dimensional torus. Indiana Univ. Math. J., 57(3):1353-1376. MR2429095(2009h:46054)

[Knese, 2010a] Knese, G. (2010a). Polynomials with no zeros on the bidisk. Anal. PDE, 3(2):109149. MR2657451

[Knese, 2010b] Knese, G. (2010b). Rational inner functions in the Schur-Agler class of the polydisk. To appear in Publicacions Matemàtiques.

[Knese, 2010c] Knese, G. (2010c). Stable symmetric polynomials and the Schur-Agler class. Preprint.

[Kummert, 1989a] Kummert, A. (1989a). Synthesis of 3-D lossless first-order one ports with lumped elements. IEEE Trans. Circuits and Systems, 36(11):1445-1449. MR1020132

[Kummert, 1989b] Kummert, A. (1989b). Synthesis of two-dimensional lossless $m$-ports with prescribed scattering matrix. Circuits Systems Signal Process., 8(1):97-119. MR.998029 (90e:94048) 
[Lotto, 1994] Lotto, B. A. (1994). von Neumann's inequality for commuting, diagonalizable contractions. I. Proc. Amer. Math. Soc., 120(3):889-895. MR.1169881 (94e:47012)

[Megretski, 2003] Megretski, A. (2003). Positivity of trigonometric polynomials. In Decision and Control, 2003. Proceedings of 42nd IEEE Conference on Decision and Control, volume 4, IEEE, pages $3814-3817$.

[Quillen, 1968] Quillen, D. G. (1968). On the representation of hermitian forms as sums of squares. Invent. Math., 5:237-242. MR0233770 (38:2091)

[Rudin, 1963] Rudin, W. (1963). The extension problem for positive-definite functions. Illinois J. Math., 7:532-539. MR0151796 (27:1779)

[Rudin, 1969] Rudin, W. (1969). Function theory in polydiscs. W. A. Benjamin, Inc., New YorkAmsterdam. MR0255841 (41:501)

[Varopoulos, 1974] Varopoulos, N. T. (1974). On an inequality of von Neumann and an application of the metric theory of tensor products to operators theory. J. Functional Analysis, 16:83-100. MR0355642 (50:8116)

Department of Mathematics, Box 870350, University of Alabama, Tuscaloosa, AlaBAMA 35487-0350

E-mail address: geknese@bama.ua.edu 